\title{
Late cardiac effect of anthracycline therapy in physically active breast cancer survivors - a prospective study
}

\author{
A. C. NAGY ${ }^{1, *}$, P. GULACSI-BARDOS ${ }^{1}$, Z. CSEREP ${ }^{1}$, L. HANGODY ${ }^{2}$, T. FORSTER ${ }^{3}$ \\ ${ }^{1}$ Uzsoki Hospital, 1st Department of Internal Medicine and Cardiology, Budapest; ${ }^{2}$ Peterfy Sandor Hospital's Traumatology Center and Uzsoki \\ Hospital's Orthopaedic and Trauma Department, Department of Traumatology, Semmelweis University, Budapest; ${ }^{3}$ University of Szeged, Faculty of \\ Medicine, Albert Szent-Gyorgyi Medical and Pharmaceutical Centre, 2nd Department of Internal Medicine and Cardiological Centre, Szeged
}

*Correspondence: nagycsaba@uzsoki.hu

Received February 12, 2016 / Accepted August 12, 2016

\begin{abstract}
The late-onset cardiotoxic effect of anthracycline is known, however the early detection and prevention of subclinical myocardial damage has not been fully understood yet. Besides medical therapy regular physical activities may also play a role in the prevention and reduction of side effects of chemotherapy. The aim of our present study was to detect the effect of regular physical activities on the diastolic function and on the symptoms of late heart failure in case of anthracycline chemotherapy. The prospective study included 55 female patients (age 31-65 year, average 49.5 years) with breast cancer and no cardiovascular risk factors. Proper cardiologic checkup included physical examination (blood pressure, pulse, etc.), ECG, standard echocardiography parameters (EF, LV dimensions etc.) and specific tissue Doppler (TDI) measurements. Symptoms of heart failure were also recorded. After five years of follow-up, symptoms of heart failure were evaluated again. Patients were assigned into two groups depending on their physical activity: 36 patients did perform regular physical activities (mean age 49.2 years) and 19 patients did not (average age 50.1 years). There was no significant difference between the two groups in basic physiological or standard echocardiography parameters neither at the baseline nor at the later time points. Diastolic dysfunction (decreased E/A) was detected 6 months after the beginning of the treatment (T2 time point) in both groups. In the inactive group this value fell below one however there was no significant difference $(1.1 \pm 0.25$ vs. $0.95 \pm 0.22)$. One year after the beginning of the treatment (T3) a significant difference could be detected between the two groups (1.05 \pm 0.28 vs. $0.86 \pm 0.25 . \mathrm{P}=0.038)$. Consistent change in diastolic function $(\mathrm{Ea} / \mathrm{Aa})$ could be detected with the more sensitive TDI (Tissue Doppler Imaging) measurements after treatments in both groups, especially in the septal segment (in the non active group the Ea/Aa decreased markedly but not significantly at T2 $-1.1 \pm 0.55$ vs. $0.81 \pm 0.44$, and this difference became significant at T3 and 2 years after treatment (T4), $\mathrm{p}=0.007$ and $\mathrm{p}=0.065)$. The filling pressure $(\mathrm{E} / \mathrm{Ea})$ rose above $10(\mathrm{p}=0.09)$ in the non active group at T2; and it kept rising in both groups and became significant at T3 ( $\mathrm{p}=0.012)$. Five years after the onset of the treatment symptoms of heart failure were less frequently reported in the physically active group than in the inactive one (19.45\% vs. $68.42 \%)$. The data of our study show that the diastolic dysfunction of the left ventricle related to the anthracycline therapy became evident in the physically active group later and the symptoms of heart failure were less frequent than in the non active group after five years period. Enrollment in sport activities could be a good means for partial prevention in this group of patients. Cardiologic checkup at proper intervals plays a pivotal role in detection of possible cardiotoxicity. This is a strong indication for changes in the lifestyle of the patient and the treatment protocol alike.
\end{abstract}

Key words: anthracycline cardiotoxicity, subclinical injury, physical activity

Anthracyclines such as epirubicin and doxorubicin are effective antineoplastic drugs effectively used in adjuvant chemotherapy used for adults and children in a wide range of malignant diseases including breast cancer, childhood tumours, soft tissue sarcomas, lymphomas and different types of leukemia. The clinical use of this highly effective anticancer drug is limited due to a severe, dose-dependent cardiotoxic effect characterized by acute cardiac injury that may progress to irreversible cardiomyopathy and congestive heart failure months even years following administration.

Anthracycline induced cardiotoxicity may have an acute, subacute or chronic manifestation [1]. With the novel 
chemotherapy protocols used in the past years (reduced dose, prolonged administration), the incidence of acute and subacute myocardial damage has significantly decreased [2]. The chronic manifestation has two well-distinguished groups, the early - and the late-onset of the disease. The former arises within one year after completing chemotherapy, whereas the latter may develop even decades later. It is characterized by a slow onset, often with intact systolic left ventricular function, and it is asymptomatic for a long time. Many factors increase the risk of its occurrence and the severity of the clinical course. These factors include age (old and very young people), the female gender, mediastinal irradiation, existing cardiovascular risk, and the cumulative dose of the drug and the frequency of its administration. The classical findings and the proof of the relationship between the risk factors and the chemotherapy were analyzed in an earlier study [3]. Cardiotoxicity depends on the cumulative dose administered. The incidence of the anthracycline-based chemotherapy induced cardiotoxicity is $0.14 \%$ in case of a cumulative dose no more than $399 \mathrm{mg} / \mathrm{m}^{2}$ is given, and reaches $3 \%$ if over a $400 \mathrm{mg} / \mathrm{m}^{2}$ dosage is administered. Over a limit of $550 \mathrm{mg} / \mathrm{m}^{2}$, it stands at $7 \%$, and with $700 \mathrm{mg} / \mathrm{m}^{2}$ it meets $18 \%$, and above $1 \mathrm{~g} / \mathrm{m}^{2}$, the rate of cardiotoxicity hits $50 \%$ or more [4]. Based on the data in the recent literature, the rate of late-onset damage is increasing [5].

Pulsatile and coloured tissue Doppler echocardiography is suitable for measuring cardiotoxic cardiomyopathy by depicting the shortening and relaxation velocities of the muscle fibres segmentally. This method is suitable to establish the diagnosis or for the follow-up. The real diagnostic value in case of diastolic dysfunction has been proofed $[6,7,8]$, compared to traditional methods [9] or to other imaging techniques [10].

There are evidences accumulating to proof that regular physical activity is an effective strategy for cancer prevention. In case of breast cancer, physical activity has been well documented to reduce cancer risk by almost $50 \%$. Exercise training prior to treatment with doxorubicin attenuates doxorubicin -induced cardiac dysfunction by maintaining fractional shortening, developed pressure, and cardiac contractility. Exercise can improve cardiovascular efficiency, increase cardiac output and stroke volume, decrease resting heart rate, lower exercise heart rate, and improve ventilation and transport of oxygen from the environment to the cell [11]. Animal studies have shown that both acute and chronic exercises are protective against doxorubicin-induced heart damage due to the increase in exercise-induced antioxidant capacity related to increased oxygen consumption. [12, 13].

The aim of the present study is to evaluate the effect of regular physical work out -prior and during the chemotherapy - on the cardiac dysfunction (diastolic dysfunction) and the development of signs of heart failure in young breast cancer patients with no cardiovascular risk. In our previously published studies, we demonstrated a decrease in the diastolic function during and after chemotherapy in this group of patients we confirmed that TDI is a suitable method for the early detection of diastolic dysfunction [14]. With reference to the above cited results we studied at this time by the help of TDI whether the preventive effect of the physical exercise already confirmed can be detected with monitoring the diastolic dysfunction.

\section{Patients and methods}

Study population. The study included 55 women, age 31-65 years, diagnosed with and operated on for right-sided malignant breast cancer and treated in the Centre for Oncoradiology, Uzsoki Hospital (Budapest, Hungary). Patients were chemotherapy naive, and had no cardiovascular risk factors previously (hypertension, diabetes mellitus, smoking, lipid disorder, obesity, etc.). Because of the right sided localization of the breast tumor the heart and the great vessels were not at risk by irradiation. Patients with low or medium risk of invasive breast cancer (stage IB, IIA, IIB - breast cancer is staged using the American Joint Committee on Cancer (AJCC) system) underwent postoperative chemotherapy, consisting of 6 series of epirubicin + cyclophosphamide or adriamycin + cyclophosphamide infusions. The cumulative dose of the doxorubicin was $240 \mathrm{mg} / \mathrm{m}^{2}$, and the cumulative dose of the epirubicin was $360 \mathrm{mg} / \mathrm{m}^{2}$. The dose of cyclophosphamide used in the study was $500 \mathrm{mg} / \mathrm{m}^{2}$ according to the standard protocol. Supportive therapy was not an exclusion criterion (e.g. painkillers, antiemetics, palliative irradiation in other localisation and bisphosphonates). Patients were assigned into two groups depending on their physical activity prior to the diagnosis. Physical activity was defined as intensive exercise program lasting at least 30 minutes at least 4-5 times a week, including any individual or team sports. Accordingly 36 patients were recruited into the physically active (PA) and 19 patients into the non-active (PNA) group.

Cardiologic examination. Cardiological history, findings of physical examinations, especially of the cardiovascular condition were recorded. A standard 12-lead electrocardiogram (ECG) was recorded, systolic and diastolic blood pressure and heart rate were measured at rest.

Standard echocardiography. The echocardiograms were performed with Vivid 3 echocardiograph (Vivid 3, GE Medical System, Horten, Norway). M-mode recordings were performed in parasternal long-axis view at the level under the apex of the mitral valves. Standard parameters were measured during systole and diastole: left ventricular dimensions, diameter of the ascending aorta and size of the left atrium. From the apical 4-chamber view the left ventricular ejection fraction was calculated according to the modified Simpson's rule, the atrial dimensions and mitral inflow velocities were measured as published previously [14]. The following parameters were derived: peak early (mitral E velocity) and atrial (mitral A velocity) flow velocities, E/A ratio, deceleration time. Pulmonary venous flow velocities were recorded. The pulmonary venous peak systolic (pulmonary S) and peak diastolic (pulmonary D) flow velocities, $S / D$ ratio, peak reverse flow velocity and duration were recorded. Ideally, the diastolic function can be 
estimated based on these data. Wall motion, valve anatomy, and the occurrence of accidental pericardial effusion were examined in all views.

Tissue Doppler Imaging (TDI). The examination was performed in TDI mode of the Vivid 3 echocardiograph. The mitral annular velocities were measured with pulsedwave TDI via transthoracic approach. Six different segments were measured (septal, posterior, septal' - septum on the parasternal long axis view, anterior, inferior and lateral) [15]. Velocity measurements were performed in 3 heart cycles and mean values of the three measurements were used in statistical analysis. From the apical 4-chamber view, four different sites of the mitral anulus were selected. Three peak velocities were recorded during TDI measurements: the positive systolic velocity ( $\mathrm{S}$ velocity), when the mitral ring moves toward the cardiac apex, and 2 negative diastolic velocities, when the mitral annulus moves toward the base away from the apex. The last two represent the early phase (Ea diast. vel.) and the late phase of diastolic (Aa diast. vel.) velocities. They were used in Ea/Aa calculations, which is a sensitive marker of diastolic dysfunction [14]. In our present study an Ea/Aa ratio below one was considered pathological. This is an artificial setup, but characteristic for diastolic dysfunction.

Left atrial filling pressure. The E/e' ratio, which represents the left atrial filling pressure, can be measured using the two methods mentioned beforehand. According to our current knowledge, a ratio below 8 safely excludes, whereas a value above 15 clearly confirms elevated filling pressure assigned to the worsening of myocardial relaxation, i.e., by diastolic failure. In-between there is the grey zone, with the need of further investigations [16].

Schedule of assessments. In our prospective study, the cardiac assessment was performed in four steps: prior to the initial chemotherapy (T1); at the halftime of the anthracycline therapy (T2); one year after the first chemotherapy (T3); 2 years after the first chemotherapy (T4). As an extended follow up, 5 years after the first assessment, the inquiry about the symptoms indicating heart failure (dyspnoea, reduced physical capacity, fatigue, foot swelling, night-time urination) was performed via of phone interviews ( $\mathrm{T} 1+5$ years).

Statistical analysis. All analyses were performed using the SPSS 13.0 statistical package (SPSS Inc, Chicago, IL). The Mann-Whitney test and Fischer's exact test were used to compare the two groups. Our results were presented as mean \pm SD.

\section{Results}

The age distribution was similar in the two groups of patients $(\mathrm{PA}-\mathrm{n}=36$ vs. $\mathrm{PNA}-\mathrm{n}=19)$ enrolled in the prospective study (49.2 vs. $50.1 \pm$ SD) (see Table 1). Applying the inclusion criteria, there were no differences in cardiovascular risk status between the two patient groups.

The baseline data of both groups are shown in Table 1. There were no appreciable differences between the two groups neither in the baseline data (T1) nor in the data at later time points. The differences observed at given time points, cannot be considered clinically relevant - although statistically significant - because both values referred to (blood pressure and pulse rate) were within normal range.

With reference to the echocardiographic characteristics there were no real differences between the groups regarding aortic diameter, left atrial and left ventricular end-diastolic diameter or the E-septum (these parameters are therefore not included in the table). Within the PNA group, the left ventricular systolic function showed a significant change (EF T3 vs. T2) but it always remained above $60 \%$ and did not cause clinical symptoms (see Table 2).

With regard to the more important diastolic function, evaluable changes were observed during the study time. The value calculated from the two waves of pulmonary inflow $(\mathrm{S} / \mathrm{D})$-, a parameter featuring diastolic function - showed an unequivocal decrease over time in both groups, thus indicating an impaired diastolic function. The differences between the two groups were not significant, the values decreased below 1 at T3 point but became significant at T4 in both groups. The evaluation of the mitral inflow waves showed unequivocally the change in diastolic function. In both groups there was a visible decrease already at $\mathrm{T} 2$, when the value fell below 1 in the PNA group, although there was no significant difference between the two groups yet. At T3, however, there was a significant difference both between the two groups and compared to T1. In the PA group, E/A decreased below 1 only at T4 (Figure 1).

Data collected by measuring different segments of the left ventricle provide an even more accurate assessment of the

Table 1. Demographic data of the patients ${ }^{1}$

\begin{tabular}{|c|c|c|c|c|c|c|c|c|c|c|c|c|}
\hline & \multicolumn{3}{|c|}{$\mathrm{T} 1$} & \multicolumn{3}{|c|}{$\mathrm{T} 2$} & \multicolumn{3}{|c|}{$\mathrm{T} 3$} & \multicolumn{3}{|c|}{ T4 } \\
\hline & $\mathrm{PA}$ & PNA & $\mathrm{p}$ & $\mathrm{PA}$ & PNA & $\mathrm{p}$ & PA & PNA & $\mathrm{p}$ & $\mathrm{PA}$ & PNA & $\mathrm{p}$ \\
\hline Mean age (years) & $49.2 \pm 10.1$ & $50.1 \pm 8.5$ & & & & & & & & & & \\
\hline Systolic blood pressure $(\mathrm{mmHg})$ & $129.6 \pm 12.2^{*}$ & $129.8 \pm 18.1$ & ns & $130.5 \pm 10.6^{\#}$ & $135.8 \pm 14.3$ & ns & $124.2 \pm 8.5$ & $128.5 \pm 12.8$ & ns & $127.6 \pm 10.8^{\#}$ & $128.1 \pm 13.9$ & ns \\
\hline Diastolic blood pressure (mmHg) & $82.3 \pm 8.3$ & $78.5 \pm 8.8$ & ns & $83.7 \pm 7.1$ & $81.7 \pm 7.7$ & ns & $80.0 \pm 7.6^{\$}$ & $80.2 \pm 7.2$ & ns & $78.6 \pm 7.8^{\varsigma . x}$ & $79.8 \pm 7.9$ & ns \\
\hline Heart rate $(1 / \mathrm{min})$ & $78.0 \pm 13.1^{\#}$ & $78.1 \pm 13.0$ & ns & $78.3 \pm 11.5^{\#}$ & $79.3 \pm 10.0$ & ns & $82.5 \pm 10.9$ & $81.2 \pm 14.8$ & ns & $82.0 \pm 11.1$ & $81.1 \pm 14.3$ & ns \\
\hline
\end{tabular}

${ }^{1}$ PA: physically active. PNA: physically non-active; $\mathrm{p}$ : difference between the two groups at each measuring times; \#: $\mathrm{p}<0.05$ vs. T3 among PA patients; $\$$ : $<<0.05$ vs. T2 among PA patients; $\mathrm{x}$ : $<<0.05$ vs. T1 among PA patients 
diastolic function with different sensitivity in the detection of impairment (Table 3). In our study we observed that, the measurement of the sept and the sept' segments proved the highest sensitivity in the detection of diastolic impairment. In the PNA group, the Ea/Aa ratio decreased below 1 already at T2 in the septal segment, whereas in the sept' segment, a value below 1 could be detected in both groups at the same time point. A ratio below 1 was measured in every segment at T3 in the PNA group, whereas in the PA group, this value decreased below 1 in the sept' and sept segments only. At T4 point a value below 1 could be observed in every segment, in both groups.

The time passing by the Ea/Aa value showed a decreasing trend in every segment in both the PA and PNA groups. This decrease became significant, compared to the baseline value (T1), at T3 in both groups, and remained significant at T4. In the PA group, there was a significant decrease in $\mathrm{Ea} / \mathrm{Aa}$ ratio compared to T2 in the sept', post and ant segments also. This indicates that the diastolic function remains intact longer in the PA group.

If comparing the values of the two groups, it can be observed that, from T2, the Ea/Aa values in the PNA group are lower than those in the PA group in every segment. This difference was significant only in the septal segment, at $\mathrm{T} 3$, and a nota-

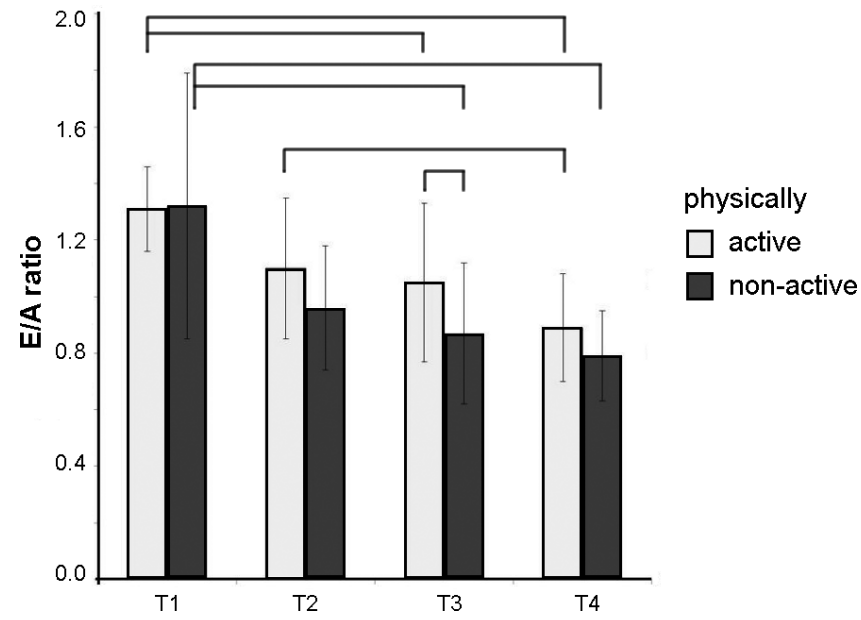

Figure 1. The E/A ratio in the physically active and non-active groups at different times. The black lines between the columns indicate significant difference $(p<0.01)$.

ble difference could be observed in the same segment at T4 $(p=0.065)$ (Figure 2). The value which is a good indicator of the left atrial filling pressure, $\mathrm{E} / \mathrm{Ea}_{\text {sept }}$, rose above 10 already at $\mathrm{T} 2 \mathrm{in}$

Table 2. Echocardiographical parameters ${ }^{1}$

\begin{tabular}{|c|c|c|c|c|c|c|c|c|c|c|c|c|}
\hline & \multicolumn{3}{|c|}{$\mathrm{T} 1$} & \multicolumn{3}{|c|}{$\mathrm{T} 2$} & \multicolumn{3}{|c|}{ T3 } & \multicolumn{3}{|c|}{$\mathrm{T} 4$} \\
\hline & PA & PNA & $\mathrm{p}$ & $\mathrm{PA}$ & PNA & $\mathrm{p}$ & PA & PNA & $\mathrm{p}$ & PA & PNA & $\mathrm{p}$ \\
\hline EF (Simpson. \%) & $66.1 \pm 5.1$ & $68.3 \pm 7.6$ & ns & $64.8 \pm 7.5$ & $65.9 \pm 5.0$ & ns & $66.2 \pm 3.4$ & $69.4 \pm 5.3^{+}$ & ns & $64.7 \pm 3.5$ & $67.8 \pm 4.4$ & 0.060 \\
\hline Mitral E velocity (m/s) & $0.76 \pm 0.16$ & $0.82 \pm 0.08$ & ns & $0.71 \pm 0.11$ & $0.69 \pm 0.12$ & ns & $0.70 \pm 0.12$ & $0.67 \pm 0.13$ & ns & $0.67 \pm 0.11$ & $0.64 \pm 0.13$ & ns \\
\hline Mitral A velocity $(\mathrm{m} / \mathrm{s})$ & $0.59 \pm 0.14$ & $0.66 \pm 0.14$ & ns & $0.66 \pm 0.10$ & $0.74 \pm 0.11$ & 0.044 & $0.69 \pm 0.10$ & $0.79 \pm 0.11$ & 0.014 & $0.76 \pm 0.09$ & $0.81 \pm 0.10$ & ns \\
\hline $\mathrm{E} / \mathrm{A}$ ratio & $1.30 \pm 0.15$ & $1.31 \pm 0.47$ & ns & $1.10 \pm 0.25$ & $0.95 \pm 0.22$ & ns & $1.05 \pm 0.28^{\#}$ & $0.86 \pm 0.25^{\star}$ & 0.038 & $0.89 \pm 0.19^{\# . \$}$ & $0.78 \pm 0.16^{*}$ & ns \\
\hline Deceleration time (ms) & $246 \pm 56$ & $264 \pm 43$ & ns & $264 \pm 55$ & $282 \pm 54$ & ns & $287 \pm 47$ & $264 \pm 64$ & ns & $299 \pm 42$ & $295 \pm 52$ & ns \\
\hline Pulmonaric venous $S(\mathrm{~m} / \mathrm{s})$ & $0.59 \pm 0.10$ & $0.54 \pm 0.10$ & ns & $0.47 \pm 0.14$ & $0.49 \pm 0.15$ & ns & $0.46 \pm 0.12$ & $0.46 \pm 0.13$ & ns & $0.44 \pm 0.12$ & $0.42 \pm 0.09$ & ns \\
\hline Pulmonaric venous $\mathrm{D}(\mathrm{m} / \mathrm{s})$ & $0.38 \pm 0.09$ & $0.34 \pm 0.08$ & ns & $0.43 \pm 0.10$ & $0.43 \pm 0.16$ & ns & $0.51 \pm 0.14$ & $0.56 \pm 0.21$ & ns & $0.53 \pm 0.11$ & $0.58 \pm 0.16$ & ns \\
\hline $\mathrm{S} / \mathrm{D}$ ratio & $1.59 \pm 0.33$ & $1.60 \pm 0.29$ & ns & $1.21 \pm 0.61$ & $1.43 \pm 0.25$ & ns & $0.98 \pm 0.40^{\#}$ & $0.94 \pm 0.51$ & ns & $0.87 \pm 0.36^{\#}$ & $0.78 \pm 0.32^{\star}$ & ns \\
\hline
\end{tabular}

${ }^{1}$ PA: physically active. PNA: physically non-active; p: difference between the two groups at each measuring times; ${ }^{*}$ : p $<0.05$ vs. T1 among PNA patients; \#: $\mathrm{p}<0.05$ vs. T1 among PA patients; +: $\mathrm{p}<0.05$ vs. T2 among PNA patients. \$: $\mathrm{p}<0.05$ vs. T2 among PA patients

Table 3. TDI parameters of the patients at different segments ${ }^{1}$

\begin{tabular}{|c|c|c|c|c|c|c|c|c|c|c|c|c|}
\hline & \multicolumn{3}{|c|}{$\mathrm{T} 1$} & \multicolumn{3}{|c|}{$\mathrm{T} 2$} & \multicolumn{3}{|c|}{$\mathrm{T} 3$} & \multicolumn{3}{|c|}{$\mathrm{T} 4$} \\
\hline & PA & PNA & $\mathrm{p}$ & PA & PNA & $\mathrm{p}$ & PA & PNA & $\mathrm{p}$ & PA & PNA & $\mathrm{p}$ \\
\hline $\mathrm{Ea} / \mathrm{Aa}$ septal & $1.63 \pm 0.23$ & $1.67 \pm 0.4$ & ns & $1.10 \pm 0.55$ & $0.81 \pm 0.44$ & ns & $0.90 \pm 0.45$ & $0.62 \pm 0.23^{*}$ & 0.007 & $0.71 \pm 0.31^{\#}$ & $0.61 \pm 0.28^{\star}$ & 0.065 \\
\hline $\mathrm{Ea} / \mathrm{Aa}$ posterior & $1.82 \pm 0.28$ & $1.81 \pm 0.53$ & ns & $1.29 \pm 0.56$ & $1.19 \pm 0.53$ & ns & $1.17 \pm 0.78^{\#}$ & $0.99 \pm 0.55^{\star}$ & ns & $0.80 \pm 0.43^{\# . S}$ & $0.81 \pm 0.45^{\star}$ & ns \\
\hline Ea/Aa septal' & $1.41 \pm 0.24$ & $1.36 \pm 0.23$ & ns & $0.97 \pm 0.42^{\#}$ & $0.84 \pm 0.35^{\&}$ & ns & $0.93 \pm 0.35^{\#}$ & $0.78 \pm 0.17^{\&}$ & ns & $0.71 \pm 0.14^{\# . s}$ & $0.63 \pm 0.07^{\star}$ & ns \\
\hline $\mathrm{Ea} / \mathrm{Aa}$ inferior & $1.52 \pm 0.32$ & $1.49 \pm 0.26$ & ns & $1.19 \pm 0.54$ & $1.01 \pm 0.41$ & ns & $1.00 \pm 0.46^{\#}$ & $0.79 \pm 0.35^{\star}$ & ns & $0.87 \pm 0.35^{\# . S}$ & $0.67 \pm 0.19^{\star}$ & ns \\
\hline $\mathrm{Ea} / \mathrm{Aa}$ lateral & $1.57 \pm 0.29$ & $1.53 \pm 0.27$ & ns & $1.39 \pm 0.61$ & $1.12 \pm 0.49$ & ns & $1.14 \pm 0.54^{\#}$ & $0.96 \pm 0.44^{*}$ & ns & $0.93 \pm 0.41^{\#}$ & $0.81 \pm 0.31^{\star}$ & ns \\
\hline $\mathrm{Ea} / \mathrm{Aa}$ anterior & $1.53 \pm 0.32$ & $1.51 \pm 0.15$ & ns & $1.18 \pm 0.55$ & $1.08 \pm 0.43$ & ns & $1.11 \pm 0.52^{\#}$ & $0.96 \pm 0.54^{*}$ & ns & $0.78 \pm 0.33^{\# . S}$ & $0.70 \pm 0.32^{*}$ & ns \\
\hline E/septE & $7.97 \pm 2.41$ & $8.70 \pm 2.71$ & ns & $9.43 \pm 2.68$ & $11.42 \pm 3.23$ & 0.09 & $10.34 \pm 2.61^{\#}$ & $12.77 \pm 3.52^{*}$ & 0.012 & $11.25 \pm 3.26^{\#}$ & $11.00 \pm 3.03$ & ns \\
\hline
\end{tabular}

${ }^{1}$ PA: physically active. PNA: physically non-active; p: difference between the two groups at each measuring times; ${ }^{*}$ : $<0.05$ vs. T1. among PNA patients; \#: $\mathrm{p}<0.05$ vs. T1 among PA patients; $\$$ : $<<0.05$ vs. T2 among PA patients; \&: $\mathrm{p}=0.059$ vs. T1 among PNA patients 


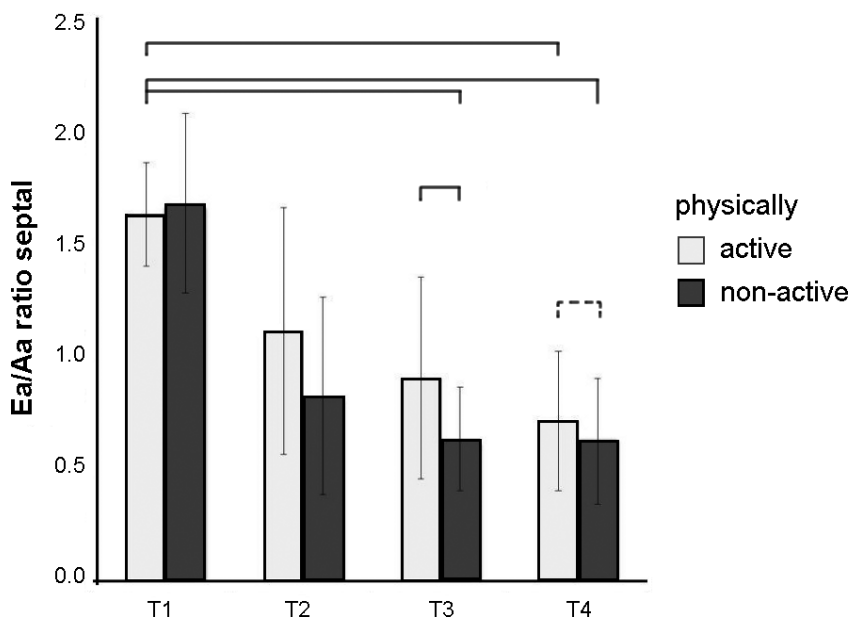

Figure 2. The Ea/Aa ratio in the physically active and non-active groups at different times. The horizontal black lines between the columns indicate significant difference $(p<0.01)$. The horizontal dashed line between the columns indicates non significant difference $(\mathrm{p}<0.065)$.

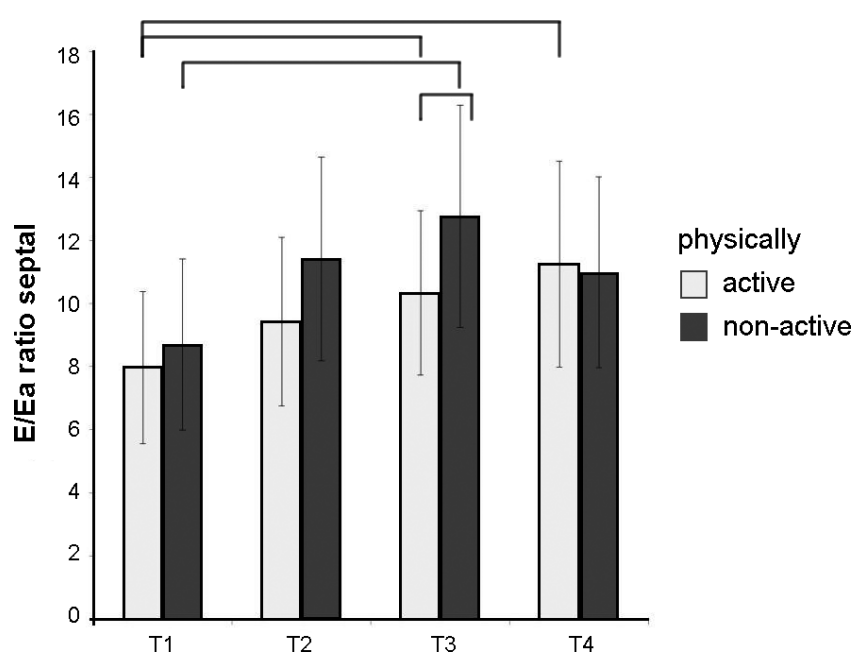

Figure 3. The E/Ea ratio in the physically active and non-active groups at different times. The black lines between the columns indicate significant difference $(\mathrm{p}<0.01)$.

the PNA group. At T3, it raised to this level in the both groups, and the value became significant compared to T1 (Figure 3 ). The increase is more accentuated in the PNA group, where it was significant compared to T2. The ratio stayed above 10 in both groups at T4. In the first two years of the study none of the patients complained of or presented with the symptoms and signs of heart failure.

Five years later, symptoms of failure were manifest (based on phone interviews) in $19.45 \%$ of the PA patients and in $68.42 \%$, (a significantly higher percentage), of the PNA patients. $80.55 \%$ of the PA patients were asymptomatic, whereas only $31.58 \%$ of the PNA patients remained symptom-free (Figure 4).

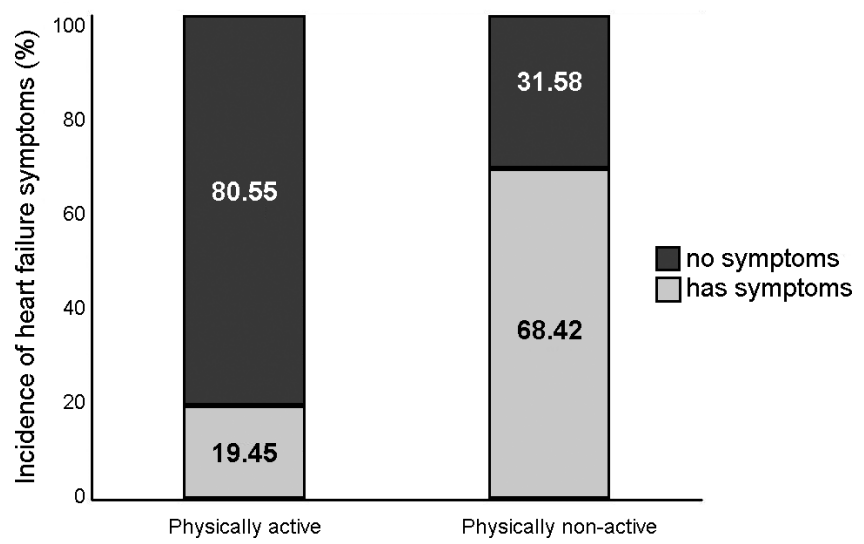

Figure 4. Incidence of heart failure symptoms 5 years after the completion of the short-term assessments. White column indicates the percentage of patients with symptoms. Black column shows the percentage of patients without symptoms.

A linear regression model was set up to confirm that sport reduces the incidence of heart failure, it was calculated by including following variables: systolic and diastolic blood pressure at the beginning, pulse rate, age and sport. In the model only sport was a significant covariate to heart failure (partial regression coefficient: $-0.51, p=0.0017$, R square 0.24 ). On this basis sport actually reduces the incidence of heart failure.

\section{Discussion}

Data collected by us confirm that in patients with a healthy heart, a diastolic dysfunction can be detected in both the PA and PNA groups after the start of chemotherapy. In the PNA group, the impairment occurred earlier. It could already be seen one year after the onset of the treatment, whereas in PA patients, it only could be observed after the second year. The difference in diastolic function between the two groups became significant after the first 1 year. When analyzing the role of the different segments, we found that the impairment is noticeable first in the septal part in the both groups. Symptoms characteristic to heart failure occurred with a significantly higher incidence in PNA patients, based on the phone interviews having been performed 5 years after completing the shortterm assessments.

By gaining more information about the cardiotoxic effect of the agents used during an aggressive chemotherapy, the treatment and the prevention - with even more emphasis - of the impairment becomes more and more important. We are familiar with the cardiotoxic effects of anthracyclines and this knowledge has been growing for decades. The entity of heart failure in children treated with doxorubicin was described already in 1967 [17].

It becomes more and more evident that with the advance of non-invasive diagnostic methods, the incidence of asymptomatic myocardial damage due to a lower cumulative dose 
of anthracycline is increasing. In addition, late-onset damage, which may develop up to decades after the therapy and is often responsible for uncontrollable heart failure, is reported with a growing frequency [18]. The best way to prevent this process is the early treatment of the subclinical myocardial damage confirmed. The first sign the altered cardiac function is the impaired diastolic function. In the past years, new methods have been sought for a more accurate determination of diastolic dysfunction. An easy-to-perform, and more and more accessible method is tissue Doppler imaging, which measures the motion velocity of a certain part of a segment (most commonly the septal or lateral one) that is close to the mitral annulus, and plots it over time. TDI indicates systolic (S wave) and diastolic (E, A wave and $\mathrm{Ea} / \mathrm{Aa}$ ) function on the basis of basal segment motion [19]. It is to be emphasized how important is the role of the mitral E/Ea ratio used to assess the left ventricular filling pressure. It helps to reveal the diastolic dysfunction by measuring the high filling pressure, regardless of systolic function. Numerous studies have been conducted on the development of diastolic dysfunction following chemotherapy $[20,21]$. It is known that the development of diastolic dysfunction precedes that of systolic dysfunction. Besides, the cardiotoxic effect may also result in isolated diastolic dysfunction, which may lead to heart failure after a long latency period. The above statements predict the importance of measuring and monitoring this parameter in the treatment of malignancies. The usefulness of the above methods for the early detection of chemotherapy-induced cardiotoxicity was confirmed in our earlier study [15].

Fifty-five young women with breast cancer were enrolled in this study. The exclusion criteria included traditional cardiovascular risk factors and irradiation of the left chest side, and therefore any change in the left ventricular function during the chemotherapy can be considered a side effect of the treatment. Patients were assessed at four time points. The echocardiography parameters measured before the start of the treatment at $\mathrm{T} 1$, were considered reference values. The rest assessment time points corresponded with the development of the subacute (T2), the early-onset chronic (T3), and the late-onset chronic (T4, and the time of the phone interview, which was 5 years after treatment) cardiotoxic effect.

The diastolic function, the value calculated from the two waves of pulmonary inflow (S/D) showed an unequivocal decrease over the time in both groups, indicating an impaired diastolic function. The differences between the two groups were not significant, the values decreased below 1 at T3 in both groups. At the same time, the difference compared to T1 became significant at $\mathrm{T} 4$ in both groups (Table 2).

The Ea/Aa value measured by TDI is considered abnormal below 1, which is an artificial limit but well characterizes the considerable impairment of diastolic function. With respect to the changes of the parameters in correspondence with the time passing by, the Ea/Aa value shows a decreasing trend in every segment in both the PA and PNA groups. This decrease became significant, compared to the baseline value (T1), at T3 point in both groups, and was still significant at T4. The study published by Di Lisi, with the aim of monitoring the diastolic function in female patients ended up with similar results: TDI was more sensitive than E/A ratio at baseline and after the chemotherapy. They also found that the reversal $\mathrm{Ea} / \mathrm{Aa}$ ratio preceded the reversal E/A ratio at baseline and after the chemotherapy, in case of diastolic dysfunction [22].

In the PA group, there was a significant decrease in the Ea/ Aa ratio compared to T2 in the sept', post and ant segments alike, which indicates that the diastolic function remains intact longer in the PA group. Measurements performed in different segments of the left ventricle provide an even more accurate assessment of the diastolic function, with different sensitivity in the detection of the impairment (Table 3). When comparing the values of the two groups, it can be observed that, from T2, the Ea/Aa values in the PNA group are lower than those in the PA group in every segment. This difference was significant only in the septal segment, at T3, and a difference showing a trend could be observed in the same segment at $\mathrm{T} 4$ $(\mathrm{p}=0.065)$ (Figure 2).

In our study, the measurement of the sept and sept' segments showed the highest sensitivity in the detection of diastolic impairment. In the PNA group, the Ea/Aa decreased below 1 already at T2 in the septal segment, whereas in the sept' segment, a value below 1 could be detected at the same time point in the both groups. At T3, a value below 1 was measured in every segment in the PNA group, whereas in the PA group, this value decreased below 1 in the sept' and sept segments only. At T4, a value below 1 could be observed in every segment, in both groups.

In the $\mathrm{PA}$ group the $\mathrm{E} / \mathrm{A}$ ratio decreased below 1 only at $\mathrm{T} 4$ (Figure 1). In the PNA group, an impaired diastolic function could be measured already at the expected time of subacute impairment (see Tables 2 and 3). Contrary to this, in the PA group, a diastolic dysfunction defined as per the ESC guideline could only be seen during the measurements performed after one year (T3-T4). Similar kinetics were observed regarding the change in the left atrial filling pressure as well. The value of $\mathrm{E} /$ $\mathrm{Ea}_{\text {sept }}$ - which is a good indicator of left atrial filling pressure - , rose above 10 already at T2 in the PNA group. At T3 point it reached this limit in both groups, and the change of the value became significant compared to T1 (Figure 3). The increase is more accentuated in the PNA group, where it was significant compared to T2. The ratio stayed above 10 in both groups at T4. A left atrial filling pressure of 8 to 15 requires further investigations to refine the diagnosis. In our study only a trend of increasing filling pressure could be detected, since no severe impairment developed that would increase the left atrial pressure considerably during the follow-up. The change in filling pressure has not yet been studied in this patient group.

With an extended follow-up (5 years after the completion of chemotherapy), significantly more cases of developing symptoms of heart failure with an impact on the quality of life and on life expectancy as well occurred among PNA patients (see Figure 4). Five years after the completion of 
the short-term assessments, performed by the help of phone interviews, symptoms characteristic to heart failure were approved in $19.45 \%$ of the PA patients and in $68.42 \%$, (a significantly higher percentage), of the PNA patients. To put it differently $80.55 \%$ of the PA patients were asymptomatic, whereas only $31.58 \%$ of the PNA patients remained symptomfree. The beneficial physiological effects of exercise and its role in reducing cardiovascular risk have been known for a long time. Numerous epidemiologic studies have confirmed the relationship between regular physical activity and the risk of developing malignancies [23]. The studies published in the past decade have provided a wide range of evidence for the stressed role of exercise in the prevention of breast cancer [24]. Beyond its role played in primary prevention, regular exercise may have an important role in secondary prevention too, the prevention of cardiotoxic side effects occurring during the chemotherapy of existing malignancies. Many experimental animal models have confirmed the protective effect of aerobic exercise against functional, histological and molecular changes caused by doxorubicin, and against the increase in mortality. Many pathophysiological factors play a role in the beneficial/ preventive effect of exercise [25]. Several human clinical studies confirm the beneficial effect of work out exercise during and after the antitumor therapy on the cardiopulmonary function and the quality of life. According to the results of Schneider et al., exercise during and after chemotherapy had a beneficial effect on the systolic and diastolic blood pressure, the heart rate and the respiratory function in patients with breast cancer. In addition, it had a positive effect on tiredness and fatigue accompanying the disease, as well as on certain sensory and cognitive functions [26]. Courneya et al. postulated a similar conclusion regarding the physical training after radio-chemotherapy in postmenopausal female patients [27].

The Framework PEACE study distinguishes between six decisive phases of tumour diseases (prescreening, screening, pretreatment, treatment, post-treatment, resumption), in all of them, the positive effect of exercise can be confirmed. During prescreening, screening phase physical activity may influence directly the cancer detection by affecting the sensitivity and specificity of the screening test and indirectly by facilitating adherence to cancer screening. The third benefit of sport is to help the prospective cancer patients build up their physical condition during the pretreatment period. During the treatment it helps patients to cope with side effects (fatigue, pain, nausea, depression etc.). After a successful treatment period physical activity is an important tool of rehabilitation [28]. Our study was conducted during and after the therapy and the benefits of exercise were observed in both cases.

Conclusions. In our previous studies, the subclinical impairment of myocardial function following anthracycline therapy in young breast cancer patients was evaluated. We confirmed that TDI is suitable for the detection of cardiotoxicity at an early stage. The impairment proved to be permanent; it was still detectable 2 years after completing the therapy [15]. Realizing the irreversible character of the impairment the highly important role of prevention must be strongly emphasized in both groups. Based upon of our result and with reference to the literature we confirm the role of physical exercise among cancer patients as an evident means in the primary and secondary prevention. In this study, we investigated whether a preventive effect of exercise can be confirmed at a stage of subclinical myocardial impairment also.

Our results show that the impairment in the diastolic function of the left ventricle (as the most sensitive marker of cardiotoxicity) had a later onset in the PA group of patients than in the PNA one. In the PA group, the incidence of any symptom of heart failure was significantly lower - even in the long term follow-up period -, compared to those patients who did not practice regular exercise (Figure 4). These results correspond with the clinical data about the beneficial preventive effects of physical exercises as described above. In our opinion, supported by the results of animal and human clinical trials (with a lower case number though) it would be favorable to implement exercise, as a widely available preventive method, at the guideline level. The method of TDI we used is easy to learn highly effective in the early detection of impairment, and it is available in every echocardiography lab. Evaluation of the TDI results does not have limitations because it is cheaper, noninvasive and shows great reproducibility. This method is important therefore for every cardiologist by providing a good means for diagnosing early cardiac impairment of a cancer patient and to help to take the necessary measures to prevent progression. Current guideline prefers the new techniques (3D echo, myocardial deformation - strain) for the clinical evidence of both types of cardiotoxicity. However, 3D echocardiography is not available at numerous centres for oncology therefore 2D Simpson remains the method for monitoring $\mathrm{EF}$ [29]. Further research is needed on a larger population to confirm the relationships found in previous studies.

Limitations. This study was conducted in a limited group of patients without cardiovascular risk factors. In the future, a prospective study with a larger sample size, longer followup and a several-year follow-up of diastolic function may be required. It is also important, during the several-year follow-up phase, to confirm the development of heart failure in the symptomatic group with a pro-BNP test, which (as a well-known marker) is an early indicator of heart failure. In addition, the new evaluation methods developing or being implemented in the meantime (cardiac MRI, strain rate, 3D echocardiography, etc.) will open a new perspectives of the more accurate assessment of diastolic function. These methods were not available at the time of our research. At the time of the study, the analysis of the pulmonary venous waveform served for the assessment of the diastolic function, although it is not included in the current guideline. Several other factors play role in the development of heart failure (diet, life style, genetic factors, etc.), but are not included in our present study.

Acknowledgments: We thank to Maria Olajos and Andrea Gondi for their assistance in assessment and data management. 


\section{References}

[1] ADAMS MJ, LIPSHULTZ SE Pathophysiology of anthracycline- and radiation-associated cardiomyopathies: implications for screening and prevention. Pediatr Blood Cancer 2005; 44: 600-606. https:/doi.org/10.1002/pbc.20352

[2] BARRY E, ALVAREZ JA, SCULLY RE, MILLER TL, LIPSHULTZ SE Anthracycline-induced cardiotoxicity: course, pathophysiology, prevention and management. Expert Opin Pharmacother 2007; 8: 1039-1058. https:/doi. org/10.1517/14656566.8.8.1039

[3] VON HOFF DD, LAYARD MW, BASA P, DAVIS HL JR, et al. Risk factors for doxorubicin-induced congestive heart failure. Ann Intern Med 1979; 91: 710-717. https:/doi. org/10.7326/0003-4819-91-5-710

[4] SWAIN SM, WHALEY FS, EWER MS Congestive heart failure in patients treated with doxorubicin: a retrospective analysis of three trials. Cancer 2003; 97: 2869-2879. https:/ doi.org/10.1002/cncr.11407

[5] MERTENS AC, YASUI Y, NEGLIA JP, POTTER JD, NESBIT $\mathrm{ME} \mathrm{JR}$ et al. Late mortality experience in five-year survivors of childhood and adolescent cancer: the Childhood Cancer Survivor Study. J Clin Oncol 2001; 19: 3163-3172.

[6] PELA G, BRUSCHI G, CAVATORTA A, MANCA C, CABASSI A et al. Doppler tissue echocardiography: myocardial wall motion velocities in essential hypertension. Eur J Echocardiogr 2001; 2: 108-117. https:/doi.org/10.1053/euje.2000.0057

[7] TABATA T, OKI T, YAMADA H, ABE M, ONOSE Y et al. Subendocardial motion in hypertrophic cardiomyopathy: assessment from long- and short-axis views by pulsed tissue Doppler imaging. J Am Soc Echocardiogr 2000; 13: 108-115. https:/doi.org/10.1016/S0894-7317(00)90021-X

[8] DANDEL M, HUMMEL M, MULLER J, WELLNHOFER E, MEYER R et al. Reliability of tissue Doppler wall motion monitoring after heart transplantation for replacement of invasive routine screenings by optimally timed cardiac biopsies and catheterizations. Circulation 2001; 104: I184-191. https:/ doi.org/10.1161/hc37t1.094855

[9] DOLZHENKO MN, RUDENKO SA, POTASHEV SV, SIMAGINA TV, NOSENKO NN et al. Left ventricle diastolic function in the patients after coronary arteries bypass graft combined with left ventricle aneurismectomy according to tissue doppler imaging: one year follow-up. Postgrad Med J 2007; 83: 320-324. https:/doi.org/10.1136/pgmj.2006.053553

[10] MONSUEZ JJ Detection and prevention of cardiac complications of cancer chemotherapy. Arch Cardiovasc Dis 2012; 105: 593-604. https:/doi.org/10.1016/j.acvd.2012.04.008

[11] SCHNEIDER C, DENNEHY C, CARTER S Exercise and Cancer Recovery. 1st ed. Champaign, Ill: Human Kinetics Publishers; 2003.

[12] ASCENSAO A, MAGALHAES J, SOARES J, FERREIRA $\mathrm{R}$, NEUPARTH $\mathrm{M}$ et al. Endurance training attenuates doxorubicin-induced cardiac oxidative damage in mice. Int J Cardiol 2005; 100: 451-460. https:/doi.org/10.1016/j. ijcard.2004.11.004

[13] CHICCO AJ, SCHNEIDER CM, HAYWARD R Voluntary exercise protects against acute doxorubicin cardiotoxicity in the isolated perfused rat heart. Am J Physiol Regul Integr Comp Physiol 2005; 289: R424-R431. https:/doi.org/10.1152/ ajpregu.00636.2004

[14] NAGY ACS, TOLNAY E, NAGYKALNAI T, FORSTER T Cardiotoxicity of anthracycline in young breast cancer female patients: the possibility of detection of early cardiotoxicity by TDI. Neoplasma 2006; 53: 511-517.

[15] NAGY AC, CSEREP Z, TOLNAY E, NAGYKALNAI T, FORSTER T Early diagnosis of chemotherapy-induced cardiomyopathy: a prospective tissue Doppler imaging study. Pathol Oncol Res 2008; 14: 69-77. https:/doi.org/10.1007/s12253-008 -9013-4

[16] CHAHAL NS, LIM TK, JAIN P, CHAMBERS JC, KOONER JS et al. Normative reference values for the tissue Doppler imaging parameters of left ventricular function: a populationbased study. Eur J Echocardiogr 2010; 11: 51-56. https:/doi. org/10.1093/ejechocard/jep164

[17] TAN C, TASAKA H, YU KP, MURPHY ML, KARNOFSKY DA Daunomycin, an antitumor antibiotic, in the treatment of neoplastic disease. Clinical evaluation with special reference to childhood leukemia. Cancer 1967; 20: 333-353. https:/doi.org/10.1002/1097-0142(1967)20:3<333::AIDCNCR2820200302>3.0.CO;2-K

[18] HEQUET O, LE QH, MOULLET I, PAULI E, SALLES G et al. Subclinical late cardiomyopathy after doxorubicin therapy for lymphoma in adults. J Clin Oncol 2004; 22: 1864-1871. https:/doi.org/10.1200/JCO.2004.06.033

[19] LENGYEL M, NAGY A, ZORANDI A Tissue Doppler echocardiography: a new technique to assess diastolic function. Orv Hetil 2002; 143: 333-339.

[20] BU'LOCK FA, MOTT MG, OAKHILL A, MARTIN RP Left ventricular diastolic function after anthracycline chemotherapy in childhood: relation with systolic function, symptoms, and pathophysiology. Br Heart J 1995; 73: 340-350. https:/ doi.org/10.1136/hrt.73.4.340

[21] MORI T, YANAGI N, MARUYAMA T, GONDO H, OKAMURA T et al. Left ventricular diastolic dysfunction induced by cyclophosphamide in blood stem cell transplantation. Jpn Heart J 2002; 43: 249-261. https:/doi.org/10.1536/jhj.43.249

[22] DI LISI D, BONURA F, MACAIONE F, PERITORE A, MESCHISI $M$ et al. Chemotherapy-induced cardiotoxicity: role of the tissue Doppler in the early diagnosis of left ventricular dysfunction. Anticancer Drugs 2011; 22: 468-472. https:/doi. org/10.1097/CAD.0b013e3283443704

[23] SUZUKI S, KOJIMA M, TOKUDOME S, MORI M, SAKAUCHI F et al. Effect of Physical Activity on Breast Cancer Risk: Findings of the Japan Collaborative Cohort Study. Cancer Epidemiol Biomarkers Prev 2008; 17: 3396-3401. https:/doi.org/10.1158/1055-9965.EPI-08-0497

[24] VAINIO H, KAAKS R, BIANCHINI F Weight control and physical activity in cancer prevention: international evaluation of the evidence. Eur J Cancer Prev 2002; 11: S94-100.

[25] SCOTT JM, KHAKOO A, MACKEY JR, HAYKOWSKY MJ, DOUGLAS PS et al. Modulation of anthracycline-induced cardiotoxicity by aerobic exercise in breast cancer: current evidence and underlying mechanisms. Circulation 2011; 124: 642-650. https:/doi.org/10.1161/CIRCULATIONAHA.111.021774 
[26] SCHNEIDER CM, HSIEH CC, SPROD LK, CARTER SD, HAYWARD R Effects of supervised exercise training on cardiopulmonary function and fatigue in breast cancer survivors during and after treatment. Cancer 2007; 110: 918-925. https:/ doi.org/10.1002/cncr.22862

[27] COURNEYA KS, MACKEY JR, BELL GJ, JONES LW, FIELD CJ et al. Randomized Controlled Trial of Exercise Training in Postmenopausal Breast Cancer Survivors: Cardiopulmonary and Quality of Life Outcomes. J Clin Oncol 2003; 21: 1660-1668. https:/doi.org/10.1200/JCO.2003.04.093
[28] COURNEYA KS, FRIEDENREICH CM Framework PEACE: an organizational model for examining physical exercise across the cancer experience. Ann Behav Med 2001; 23: 263-272. https:/doi.org/10.1207/S15324796ABM2304 5

[29] PLANA JC, GALDERISI M, BARAC A, EWER MS, KY B et al. Expert Consensus for Multimodality Imaging Evaluation of Adult Patients during and after Cancer Therapy: A Report from the American Society of Echocardiography and the European Association of Cardiovascular Imaging. J Am Soc Echocardiogr 2014; 27: 911-939. https:/doi.org/10.1016/j.echo.2014.07.012 